\title{
Ecology, endemism, and conservation status of birds that collide with glass windows in Monteverde, Costa Rica
}

\author{
Rose Marie Menacho-Odio ${ }^{1,2,3 *}$, Martha Garro-Cruz ${ }^{4} \&$ J. Edgardo Arévalo ${ }^{5,6}$ \\ 1. Programa de Manejo de Recursos Naturales, Escuela de Ciencias Exactas y Naturales. Universidad Estatal a \\ Distancia.474-2050, San José, Costa Rica; rmenacho@uned.ac.cr \\ 2. Asociación Ornitológica de Costa Rica. 572-1250, Escazú, Costa Rica \\ 3. Programa de Doctorado en Ciencias Naturales para el Desarrollo (DOCINADE), Universidad Nacional, 86-3000 \\ Heredia, Costa Rica \\ 4. Departamento de Investigación, Universidad de Georgia Costa Rica, Aptdo.108-10655, San Luis, Monteverde, Costa \\ Rica; ugacrheadnaturalist@gmail.com \\ 5. The School for Field Studies, Apartado Postal 150-4013, Atenas, Alajuela, Costa Rica. \\ 6. Escuela de Biología, Universidad de Costa Rica, 11501-2060 San Pedro, San José, Costa Rica; \\ jose.arevalohernandez@ucr.ac.cr \\ * Correspondence
}

Received 02-X-2018. Corrected 21-XII-2018. Accepted 17-I-2019.

\begin{abstract}
Worldwide, billions of birds die annually due to window collisions. Nevertheless, few accounts document bird-window collisions in the Neotropics. In this study, we document species that collided with windows in Monteverde, Costa Rica, and describe their ecological and conservation status. We gathered information from different sources, including data from museum records and accounts by Monteverde residents who participated as "citizen scientists" between May 2014 and December 2017. We conducted carcass searches between March 2015 and February 2016. We classified window-strike species by migratory, forest dependence, trophic guild, weight, abundance, conservation, and endemism status. We registered 103 species striking windows in Monteverde, which includes 98 of 267 species known to occur in three life zones in Monteverde and five not registered in the area. Window strike casualties' frequencies differed by species, trophic guild and migratory status. Most window victims were residents, small, insectivorous, considered common or fairly common, with declining population trends. The families with the most species represented were Parulidae (14 spp.), Trochilidae (13 spp.), Turdidae (10 spp.), and Tyrannidae (9 spp.). Most species were passerines (Order Passeriformes) (71 spp.). No hawks or vultures were found colliding with buildings. The three species most commonly killed by windows were frugivores: Swainson's Thrush (Catharus ustulatus), Northern Emerald-Toucanet (Aulacorhynchus prasinus), and Black-faced Solitaire (Myadestes melanops). Among window-kills were five species whose status on the IUCN Red List are Near Threatened and one Vulnerable, including the Resplendent Quetzal (Pharomachrus mocinno) and the Three-wattled Bellbird (Procnias tricarunculatus). Six species are listed as in danger of extinction and four are listed as species with reduced populations by the National System of Conservation Areas for Costa Rica (SINAC). 12 endemic species are strike casualties. The premontane wet forest is the life zone where more species were found ( $n=64 \mathrm{spp}$.), followed by the premontane moist forest ( $\mathrm{n}=49 \mathrm{spp}$.) and the lower montane wet forest $(\mathrm{n}=31 \mathrm{spp}$.). These findings demonstrate the urgent need for conservation measures to mitigate bird mortality due to window collisions. Promoting use of methods to protect birds from windows should be an important goal for this IBA and the rest of Costa Rica. We also recommend collecting data in order to increase understanding about bird window collisions.
\end{abstract}

Key words: avian mortality; threatened species; window strikes; life zones; collision vulnerability; citizen science; Monteverde; Tropical Cloud Forest.

Menacho-Odio, R. M., Garro-Cruz, M., \& Arévalo, J. E. (2019). Ecology, endemism, and conservation status of birds that collide with glass windows in Monteverde, Costa Rica. Revista de Biología Tropical, 67(2) Suplemento, S326-S345. 
Billions of birds are killed annually worldwide by window collisions (Klem, 2015). In the United States, the annual estimated death toll is 365 to 988 million (Loss, Will, Loss, \& Marra, 2014) and 16 to 42 million in Canada (Machtans, Wedeles, \& Bayne, 2013).Although many of these deaths are caused by collisions with high rise urban buildings (Klem, Farmer, Delacretaz, Gelb, \&S aenger, 2009; Hager \& Craig, 2014; Cusa, Jackson, \& Mesure, 2015), the majority of deaths occur on single and twostory residences in suburban and rural areas due to their proportionately larger numbers (Machtans et al., 2013; Loss et al., 2014) and surrounding habitat features, such as the presence of trees (Gelb \& Delacretaz, 2009; Klem et al., 2009; Borden \& Lockhart, 2010). Loss et al. (2014) and Machtans \& Thogmartin (2014) recommended assessing the vulnerability of specific species to window collisions in order to determine effects on their respective populations and to establish species-oriented conservation management priorities.

While many studies document bird collisions in the United States and Canada, relatively few studies have taken place in the Neotropics; these include studies from Colombia (Agudelo-Álvarez, 2006; Agudelo-Álvarez, Moreno-Velasques, \& Ocampo-Peñuela, 2010; Ocampo-Peñuela et al., 2016), Costa Rica (Graham, 1997; Menacho-Odio, 2015; Oviedo \& Menacho-Odio, 2015), Mexico (Cupul-Magaña, 2003; Gómez-Moreno, Herrera-Herrera, \& Niño-Maldonado, 2018), and Brazil (Briske, Campos-Silva, \& Piratelli, 2017; Santos, Ferreira, \& Ferreira, 2017). Studies conducted in Important Bird Areas (IBA) are even more infrequent (Santos et al., 2017).

Monteverde-Arenal is one of 21 Important Bird Areas (IBA) designated for Costa Rica (Sánchez, Criado, Sánchez, \& Sandoval, 2009). Its creation was based on three global criteria: the presence of threatened species, species with restricted range, and restricted biome (Bird Life International, 2017). Monteverde is a well-known tourist destination (Davis, 2009) where urban development has favored the use of panels of glass in the infrastructure which is often within or adjacent to forest areas (Menacho-Odio, 2018). Although Young \&McDonald (2014) stated that hundreds of birds die every year due to window collisions in Monteverde, no studies describe the species affected.

In this study, we documented species and quantified species-specific characteristics of window casualties in the highly diverse rural Monteverde area, Costa Rica (Young $\&$ McDonald, 2014). We include characteristics such as the life zones where they were found, migratory habits, forest dependence, trophic guild, weight, endemism, and conservation status of each species. Our findings provide a baseline for future studies to understand and quantify the full extent of this problem in Monteverde as well as elsewhere in Costa Rica and the Neotropics.

\section{MATERIAL AND METHODS}

Study area: We conducted this study from August 2014 to December 2017 in Monteverde, located on the Pacific slope of the Tilarán mountain range $\left(10^{\circ} 18^{\prime} \mathrm{N}, 84^{\circ} 49 \mathrm{~W}\right.$; elevation 700-1 400 m a.s.1.), Puntarenas, Costa Rica. The category "Monteverde" refers to the region that includes the community area of Monteverde, the Monteverde Cloud Forest Preserve, the Children's Eternal Rainforest, and areas on both sides of the Continental Divide down to about $700 \mathrm{~m}$ elevation (Nadkarni \& Wheelwright, 2014) (Fig. 1).

Monteverde encompasses seven life zones of the twelve found in Costa Rica (Holdridge, 1967). Our study area includes three life zones (Fogden, 2014): premontane moist forest, premontane wet forest, and lower montane wet forest (Fig. 1). The moisture gradient on the Pacific slope causes a more abrupt variation than on the Caribbean slope, resulting in high species diversity both for plants and birds in each life zone (Kappelle, 2016).

In our study area 267 bird species had been recorded by Fogden (2014), including 156 residents, 41 altitudinal migrants, 67 latitudinal migrant species, and three latitudinal migrants with reproductive populations. This list does 


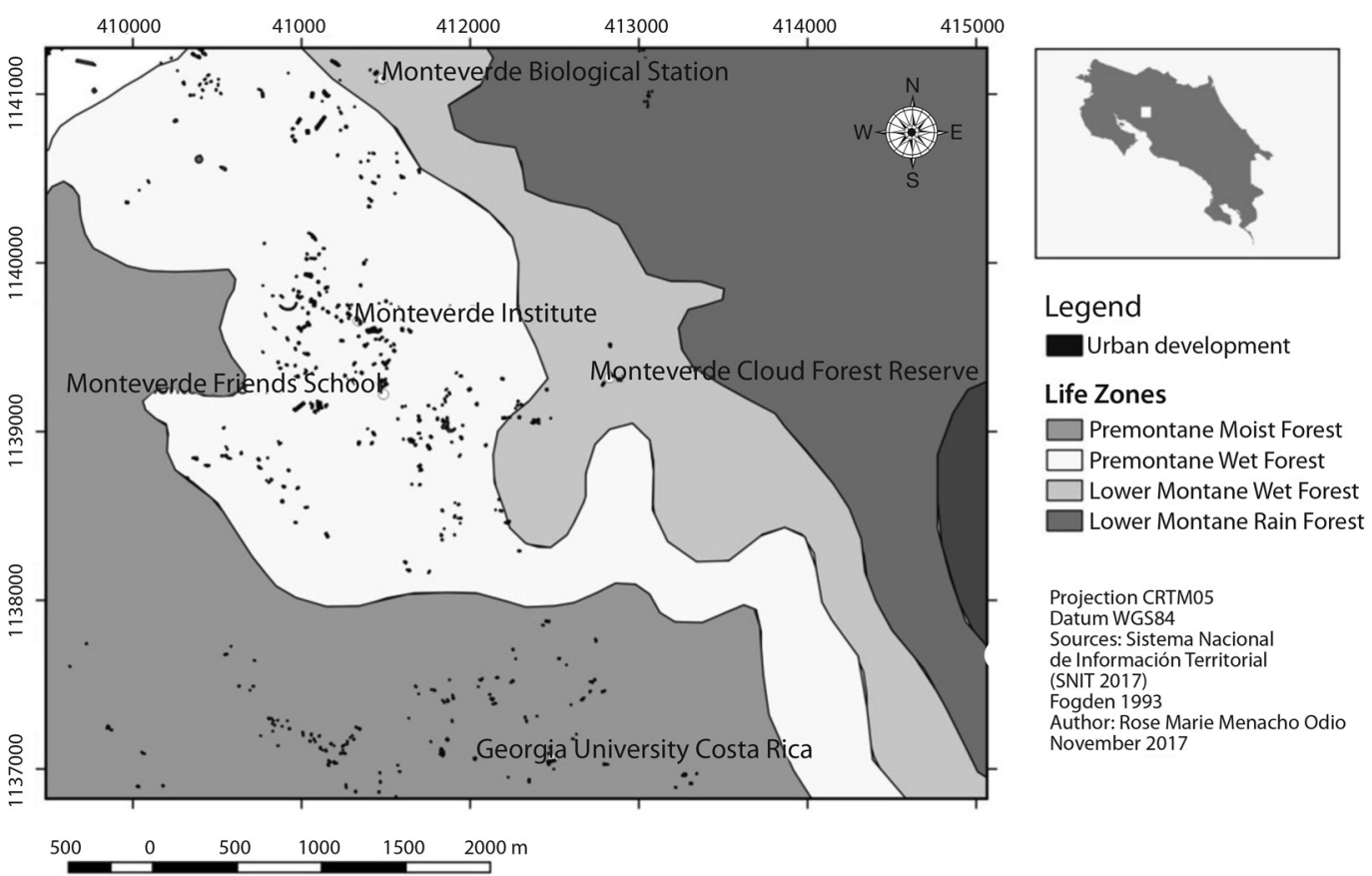

Fig. 1. Life zones and urban development of Monteverde, Costa Rica 2017 (Fogden, 2014; SNIT, 2017).

not include vagrant or uncertain species. Two species are endemic to the continental area of Costa Rica and 18 are restricted to the highlands of Costa Rica and western Panama (Obando-Calderón et al., 2017).

The populations of most bird species in Monteverde have not been estimated. Stiles \& Skutch (2003) and Garrigues (2014) provide a general idea of bird abundance. Likewise, Fogden (2014) described the abundance of the species in each Monteverde life zone. The only exception is the Three-Wattled Bellbird (Procnias tricarunculatus) for which annual counts during a six year period estimate 116-248 individuals in Monteverde (Sandoval, 2014).

Data collection: We documented window-strike species by using museum records and citizen observations, and by monitoring designated buildings (Klem, 1989). Specifically, in 2014 and 2017, we obtained from the National Museum of Costa Rica and the Museum of Zoology of the University of Costa Rica a list of specimens with tags specifying that they had collided with windows in Monteverde. We also obtained records from residents of Monteverde who submitted specimens of window-killed birds.

Carcass surveys: Buildings designated for carcass surveys were those of organizations dedicated to education, conservation, and research, as well as private homes of residents, because they were willing to collect and share data. These included the Monteverde Institute, Monteverde Friends School, and a private home located in premontane wet forest. In lower montane wet forest we visited the Monteverde Cloud Forest Reserve, the Monteverde Biological Station, and one private building. The private residences were chosen because the residents of one of them reported high window-strike mortality and the other one because it had large panes of glass. All the buildings are one or two stories, $2.5 \mathrm{~m}$ to $20 \mathrm{~m}$ height, and surrounded by secondary forest and gardens. Another criterion was that the buildings were close enough together for 
the main researcher (RMMO) to visit during a day in order to do a carcass search. In all cases, we obtained permission from property owners (Fig. 1). From March 2015 to February 2016, RMMO conducted surveys of carcasses eight days per month, with the exception of four days in April and seven days in September and February, for a total of 90 sampling days per buildings. She conducted surveys around 27 buildings including the Monteverde Institute ( $\mathrm{n}$ $=5$ buildings), Monteverde Biological Station $(\mathrm{n}=7)$, Monteverde Cloud Forest Reserve (n $=8)$, Friends School $(\mathrm{n}=5)$, and two houses belonging to area residents. The protocol consisted in looking for carcasses around each building on a transect of two meters width from the building's wall (Hager \& Cosentino, 2014). She collected all the carcasses, taking pictures of the bird and the window where she judged it had hit.

From October 2014 to March 2016, we asked inhabitants of Monteverde and the staff of the collaborating organizations to collect bodies of birds killed by window collisions. We made this request through electronic media, such as the newsletters of the Monteverde Conservation League, the Monteverde Institute, and the Friends School, and through signs posted in the area, and personal visits to community meetings. The carcasses were stored in freezers at the Monteverde Institute, the Monteverde Biological Station, and the Monteverde Cloud Forest Reserve laboratory. Following this study, we delivered most of the bodies to the Natural History Department of the National Museum of Costa Rica. These specimens are registered with the following catalogue numbers: MNCR-O28910, MNCR-O28912, MNCRO28913， MNCR-O28915, MNCR-O28916, MNCR-O28918, MNCR-O28920, MNCRO28921, MNCR-O28923, and MNCR-O28927 (G. Alvarado, pers. comm., February 20, 2018).

Photo reports: Through social media, we also received from Monteverde residents photo reports of birds, dead or alive, that collided with windows. Photographs from bird guides were used to identify the species (Kummer,
Bayne, \& Machtans, 2016). From March 2015 to December 2017, we received and took photographs of birds that had collided with windows at the University of Georgia Costa Rica campus in San Luis Valley, located in premontane moist forest (Fig. 1).

Naturalists: From August 2014 to December 2017, we received specimens of birds that were collected and frozen by local biologists and residents. Each specimen was identified, a photo was taken, and the date and place where it was found were registered.

Expert interviews: From August to November 2014, we interviewed five biologists who have lived in Monteverde for more than ten years. We asked them to identify the species they were aware of striking windows most frequently.

Community survey: During 18-22 February 2016, with the collaboration of two volunteers, we interviewed 58 Monteverde residents aged 15 and older. One survey was conducted per household. The Monteverde community area was divided into seven conglomerates with an average of 20 houses, and RMMO and two volunteers visited each conglomerate during half of a day, aiming to visit as many houses as possible. The survey questionnaire asked: Has a bird ever died when it hit a window in this house? The people who answered affirmatively were asked: What species have hit the house? A list of species was obtained and the frequency of strike records per species was collected. Additionally, residents were shown eight pictures with names: Green Hermit (Phaethornis guy), Swainson's Thrush (Catharus ustulatus), Three-wattled Bellbird, Resplendent Quetzal (Pharomachrus mocinno), Long-tailed Manakin (Chiroxiphia linearis), White-tipped Dove (Leptotila verreauxi), Lesson's Motmot (Momotus lessonii), and Northern EmeraldToucanet (Aulacorhynchus prasinus). We asked each person: Which of these birds died colliding with windows? We categorized the answers 
as "yes", "no", "I don't know" or "no answer" for each picture.

Data analysis: We identified each specimen and designated the casualties according to categories: 1) Migratory status (Fogden, 2014): residents, altitudinal migrants, latitudinal migrants, and breeding resident with latitudinal migration populations; 2) Forest dependence (Stiles \& Skutch, 2003; Biamonte, Sandoval, Chacón, \& Barrantes, 2011): 1= species that live in mature forests, $2=$ species that live in habitats with $50 \%$ or less forest covers, and $3=$ species that live in open areas. Species between a-b and c-d where classified as 1.5 and 2.5 using data from Stiles \& Skutch (2003); 3) Feeding guilds (Stiles \& Skutch, 2003; González-Salazar, Martínez-Meyer, \& López-Santiago,2014): frugivore, insectivore, granivore, nectarivore, carnivore, aquatic invertebrates eaters, omnivores, and scavengers; 4) Weight (Stiles \&S kutch, 2003); 5) Abundance (Fogden, 2014): Common, Fairly common, Uncommon, Rare,
Status Uncertain, Vagrant, and not reported in the zone;6) Endemism (Garrigues et al., 2017): Endemics to continental Costa Rica, Endemics to highlands of Costa Rica and western Panama, Endemics to Caribbean, Honduras to Panama, and Endemics to Eastern Honduras to western Panama; 7) Conservation and population trend status (IUCN, 2017; SINAC, 2017).

Carcasses collected were compared by age, sex, and collision frequency per month. To determine the species that collided most frequently, we used records from interviews with biologists, specimens collected, and the area surveys.

\section{RESULTS}

Number of species: We recorded 103 species, classified in 25 families and eight orders, that collided with windows in Monteverde (Table 1). $69 \%$ (71 species) belonged to the order Passeriformes. We recorded 98 species (36.7\%) of the 267 species documented and five species

Taxonomic classification, life zones, migratory status, forest dependence, trophic guild, endemism, and conservation status of birds that collided with windows in Monteverde, Costa Rica. Data obtained from August 2014 to December 2017

\begin{tabular}{|c|c|c|c|c|c|c|c|c|}
\hline Taxon $^{1}$ & 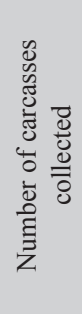 & 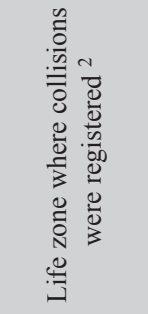 & 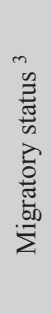 & 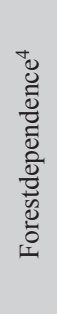 & 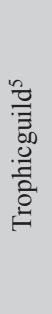 & 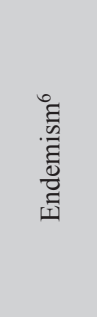 & 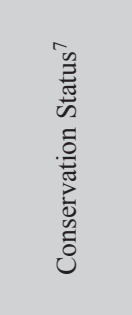 & 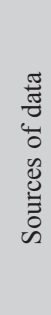 \\
\hline \multicolumn{9}{|l|}{ Cracidae } \\
\hline $\begin{array}{l}\text { Gray-headed Chachalaca } \\
\text { Ortalis cinereiceps }\end{array}$ & 1 & PMF, PWF & $\mathrm{R}$ & 3 & $\mathrm{~F}$ & & $\mathrm{LC}^{\mathrm{D}}$ & MR \\
\hline $\begin{array}{l}\text { Black Guan } \\
\text { Chamaepetes unicolor }\end{array}$ & 1 & PWF & $\mathrm{R}$ & 1.5 & $\mathrm{~F}$ & CRWP & $\mathrm{NT}^{\mathrm{D}} /$ End & MR \\
\hline \multicolumn{9}{|l|}{ Columbidae } \\
\hline $\begin{array}{l}\text { Red-billedPigeon } \\
\text { Patagioenas flavirostris }\end{array}$ & 0 & PWF & $\mathrm{R}$ & 3 & $\mathrm{~F}$ & & $\mathrm{LC}^{\mathrm{D}}$ & MR \\
\hline $\begin{array}{l}\text { Band-tailed Pigeon } \\
\text { Patagioenas fasciata }\end{array}$ & 0 & PWF & $\mathrm{E}$ & 2 & G & & $\mathrm{LC}^{\mathrm{D}}$ & MR \\
\hline $\begin{array}{l}\text { Short-billed Pigeon } \\
\text { Patagioenas nigrirostris }\end{array}$ & 0 & PMF & $\mathrm{E}$ & 2 & $\mathrm{~F}$ & & $\mathrm{LC}^{\mathrm{S}}$ & MR \\
\hline
\end{tabular}




\begin{tabular}{|c|c|c|c|c|c|c|c|c|}
\hline \multicolumn{9}{|c|}{ CUADRO 2 (Continuación) / TABLE 2 (Continued) } \\
\hline $\begin{array}{l}\text { Inca Dove } \\
\text { Columbina inca }\end{array}$ & 0 & PWF & $\mathrm{R}$ & 3 & G & & $\mathrm{LC}^{\mathrm{I}}$ & MR \\
\hline $\begin{array}{l}\text { Ruddy Quail-Dove } \\
\text { Geotrygon montana }\end{array}$ & 1 & PMF, PWF & $\mathrm{R}$ & 1.5 & G & & $\mathrm{LC}^{\mathrm{D}}$ & MR \\
\hline $\begin{array}{l}\text { White-tipped Dove } \\
\text { Leptotila verreauxi }\end{array}$ & 1 & PMF, PWF & $\mathrm{R}$ & 2.5 & G & & $\mathrm{LC}^{\mathrm{I}}$ & $\mathrm{M}, \mathrm{SC}, \mathrm{MR}$ \\
\hline $\begin{array}{l}\text { ChiriquiQuail-Dove } \\
\text { Zentrygon chiriquensis }\end{array}$ & 1 & LMWF & $\mathrm{R}$ & 1.5 & G & CRWP & $\mathrm{LC}^{\mathrm{S}}$ & MR \\
\hline \multicolumn{9}{|l|}{ Cuculidae } \\
\hline $\begin{array}{l}\text { Squirrel Cuckoo } \\
\text { Piaya cayana }\end{array}$ & 0 & PMF & $\mathrm{R}$ & 2.5 & I & & $\mathrm{LC}^{\mathrm{S}}$ & MR \\
\hline $\begin{array}{l}\text { Mangrove Cuckoo } \\
\text { Coccyzus minor }\end{array}$ & 0 & PWF & $\mathrm{L}$ & 2 & I & & $\mathrm{LC}^{\mathrm{S} /} \mathrm{RPT}$ & MR \\
\hline \multicolumn{9}{|l|}{ Apodidae } \\
\hline $\begin{array}{l}\text { Vaux's Swift } \\
\text { Chaetura vauxi }\end{array}$ & 0 & PMF & $\mathrm{R}$ & 2 & I & & $\mathrm{LC}^{\mathrm{I}}$ & MR \\
\hline \multicolumn{9}{|l|}{ Trochilidae } \\
\hline $\begin{array}{l}\text { White-necked Jacobin } \\
\text { Florisuga mellivora }\end{array}$ & 0 & PWF & E & 2 & $\mathrm{~N}$ & & $\mathrm{LC}^{\mathrm{U}}$ & MR \\
\hline $\begin{array}{l}\text { Green Hermit } \\
\text { Phaethornis guy }\end{array}$ & 3 & PMF, PWF & E & 1.5 & $\mathrm{~N}$ & & $\mathrm{LC}^{\mathrm{U}}$ & MR \\
\hline $\begin{array}{l}\text { Green-crowned Brilliant } \\
\text { Heliodoxa jacula }\end{array}$ & 1 & LMWF & $\mathrm{R}$ & 1.5 & $\mathrm{~N}$ & & $\mathrm{LC}^{\mathrm{D}}$ & MR \\
\hline $\begin{array}{l}\text { Plain-capped Starthroat } \\
\text { Heliomaster constantii }\end{array}$ & 0 & PMF & $\mathrm{R}$ & 2.5 & $\mathrm{~N}$ & & $\mathrm{LC}^{\mathrm{S}}$ & MR \\
\hline $\begin{array}{l}\text { Purple-throated Mountain-gem } \\
\text { Lampornis calolaemus }\end{array}$ & 2 & PWF,LMWF & $\mathrm{R}$ & 2 & $\mathrm{~N}$ & CRWP & $\mathrm{LC}^{\mathrm{S}}$ & $\mathrm{M}, \mathrm{MR}$ \\
\hline $\begin{array}{l}\text { Magenta-throated Woodstar } \\
\text { Calliphlox bryantae }\end{array}$ & 0 & LMWF & $\mathrm{R}$ & 2.5 & $\mathrm{~N}$ & CRWP & $\mathrm{LC}^{\mathrm{S}}$ & MR \\
\hline $\begin{array}{l}\text { Ruby-throated Hummingbird } \\
\text { Archilochus colubris }\end{array}$ & 5 & PWF & $\mathrm{L}$ & 3 & $\mathrm{~N}$ & & $\mathrm{LC}^{\mathrm{I}}$ & MR \\
\hline $\begin{array}{l}\text { Canivet's Emerald } \\
\text { Chlorostilbon canivetii }\end{array}$ & 0 & PMF & $\mathrm{R}$ & 3 & $\mathrm{~N}$ & & $\mathrm{LC}^{\mathrm{U}}$ & MR \\
\hline $\begin{array}{l}\text { Violet Sabrewing } \\
\text { Campylopterus hemileucurus }\end{array}$ & 4 & PMF, PWF,LMWF & E & 2 & $\mathrm{~N}$ & & $\mathrm{LC}^{\mathrm{U}}$ & MR \\
\hline $\begin{array}{l}\text { Stripe-tailed Hummingbird } \\
\text { Eupherusa eximia }\end{array}$ & 5 & PMF, PWF,LMWF & E & 2 & $\mathrm{~N}$ & & $\mathrm{LC}^{\mathrm{U}}$ & $\mathrm{SC}, \mathrm{MR}$ \\
\hline $\begin{array}{l}\text { Coppery-headed Emerald } \\
\text { Elvira cupreiceps }\end{array}$ & 3 & PWF & $\mathrm{E}$ & 2 & $\mathrm{~N}$ & $\mathrm{CR}$ & $\mathrm{LC}^{\mathrm{U}}$ & MR \\
\hline $\begin{array}{l}\text { Steely-vented Hummingbird } \\
\text { Amazilia saucerottei }\end{array}$ & 0 & PWF & $\mathrm{R}$ & 2.5 & $\mathrm{~N}$ & & $\mathrm{LC}^{\mathrm{I}}$ & MR \\
\hline $\begin{array}{l}\text { Blue-throated Goldentail } \\
\text { Hylocharis eliciae }\end{array}$ & 0 & PMF & $\mathrm{E}$ & 3 & $\mathrm{~N}$ & & $\mathrm{LC}^{\mathrm{I}}$ & MR \\
\hline \multicolumn{9}{|l|}{ Trogonidae } \\
\hline $\begin{array}{l}\text { Orange-bellied Trogon } \\
\text { Trogon aurantiiventris }\end{array}$ & 1 & PWF, LMWF & $\mathrm{R}$ & 1.5 & $\mathrm{~F}$ & CRWP & $\mathrm{LC}^{\mathrm{D} / \mathrm{RPT}}$ & MR \\
\hline $\begin{array}{l}\text { Resplendent Quetzal } \\
\text { Pharomachrus mocinno }\end{array}$ & 0 & LMWF & $\mathrm{E}$ & 1.5 & $\mathrm{~F}$ & & $\mathrm{NT}^{\mathrm{D}} / \mathrm{RPT}$ & MR \\
\hline \multicolumn{9}{|l|}{ Momotidae } \\
\hline $\begin{array}{l}\text { Lesson's Motmot } \\
\text { Momotus lessonii }\end{array}$ & 3 & PMF, PWF, LMWF & $\mathrm{R}$ & 2.5 & I & & $\mathrm{LC}^{\mathrm{D}}$ & MR \\
\hline
\end{tabular}


CUADRO 2 (Continuación) / TABLE 2 (Continued)

\section{Ramphastidae}

Northern Emerald-Toucanet Aulacorhynchus prasinus

Keel-billed Toucan

Ramphastos sulfuratus

\section{Psittacidae}

Brown-hooded Parrot

Pyrilia haematotis

White-fronted Parrot

Amazona albifrons

\section{Thamnophilidae}

Slaty Antwren

Myrmotherula schisticolor

\section{Grallariidae}

Scaled Antpitta

Grallaria guatimalensis

\section{Furnariidae}

Gray-throated Leaftosser

Sclerurus albigularis

Ruddy Woodcreeper

Dendrocincla homochroa

Northern-barred Woodcreeper

Dendrocolaptes sanctithomae

Spotted Woodcreeper

Xiphorhynchus erythropygius

Spotted Barbtail

Premnoplex brunnescens

\section{Tyrannidae}

Yellow-bellied Elaenia

Elaenia flavogaster

Mountain Elaenia

Elaenia frantzii

Paltry Tyrannulet

Zimmerius vilissimus

Olive-striped Flycatcher

Mionectes olivaceus

Tufted Flycatcher

Mitrephanes phaeocercus

Dusky-capped Flycatcher

Myiarchus tuberculifer

Yellow-bellied Flycatcher

Empidonax flaviventris

Yellowish Flycatcher

Empidonax flavescens

Sulphur-bellied Flycatcher

Myiodynastes luteiventris

\section{Cotingidae}

Three-wattled Bellbird

Procnias tricarunculatus

\section{Pipridae}

Long-tailed Manakin

Chiroxiphia linearis

$\begin{array}{ccccccc}14 & \text { PMF, PWF, LMWF } & \text { E } & 2 & \text { F } & \text { LC } & \text { M, SC, MR } \\ 0 & \text { PMF, PWF } & \text { R } & 2 & \text { F } & \text { LC } & \text { MR }\end{array}$

$\begin{array}{llllllll}0 & \text { PWF } & \text { R } & 1.5 & \text { F } & \text { LC } & \text { MR } \\ 0 & \text { PWF } & \text { R } & 2 & \text { F } & \text { LC } & \text { MR }\end{array}$

LMWF

R $1.5 \quad \mathrm{I}$

$\mathrm{LC}^{\mathrm{D}}$

MR

0

PWF

R 1 I

$\mathrm{LC}^{\mathrm{D}}$

M

2

LMWF

R 1 I

$\mathrm{NT}^{\mathrm{S}}$

M, SC, MR

PMF, LMWF

R 2 I

$\mathrm{LC}^{\mathrm{D}}$

MR

0

PMF

R 2 I

$\mathrm{LC}^{\mathrm{D}}$

MR

PWF

R $1.5 \quad$ I

$\mathrm{LC}^{\mathrm{S}}$

MR

0

PWF

R $1.5 \quad$ I

$\mathrm{LC}^{\mathrm{S}}$

MR

PMF

R 3 I

$\mathrm{LC}^{\mathrm{S}}$

MR

LMWF

E $2.5 \quad$ I

$\mathrm{LC}^{\mathrm{S}}$

M, MR

1

LMWF

E 3 I

$\mathrm{LC}^{\mathrm{S}}$

MR

10

PMF, PWF,LMWF

E $1.5 \quad$ I

$\mathrm{LC}^{\mathrm{S}}$

M, SC, MR

1

PWF

R 2 I

$\mathrm{LC}^{\mathrm{S}}$

MR

0

PWF

$\begin{array}{lll}\text { R } & 2.5 \quad \mathrm{I}\end{array}$

$\mathrm{LC}^{\mathrm{D}}$

MR

1

PMF, PWF

L 2 I

$\mathrm{LC}^{\mathrm{I}}$

MR

1

PWF

R 2 I

$\mathrm{LC}^{\mathrm{S}}$

SC, MR

$0 \quad$ PMF

$\begin{array}{ccc}\text { RR, } & 2.5 & \mathrm{I}\end{array}$

$\mathrm{LC}^{\mathrm{S}}$

MR

0

PWF

E $\quad 1.5 \quad$ F $\quad$ EHWP $\quad V^{\mathrm{D}} /$ End

M, MR 
CUADRO 2 (Continuación) / TABLE 2 (Continued)

\section{Vireonidae}

Lesser Greenlet

Pachysylvia decurtata

Yellow-throated Vireo

Vireo flavifrons

Philadelphia Vireo

Vireo philadelphicus

Red-eyed Vireo

Vireo olivaceus

Yellow-green Vireo

Vireo flavoviridis

Troglodytidae

House Wren

Troglodytes aedon

Rufous-breasted Wren

Pheugopedius rutilus

Rufous-and-white Wren

Thryophilus rufalbus

Cabanis's Wren

Cantorchilus modestus

\section{Turdidae}

Black-faced Solitaire

Myadestes melanops

Orange-billed Nightingale

Catharus aurantiirostris

Slaty-backed Nightingale-Thrush

Catharus fuscater

Black-headed Nightingale

Catharus mexicanus

Gray-cheeked Thrush

Catharus minimus

Swainson's Thrush

Catharus ustulatus

Wood Thrush

Hylocichla mustelina

Mountain Thrush

Turdus plebejus

Clay-colored Thrush

Turdus grayi

White-throated Thrush

Turdus assimilis

\section{Bombycillidae}

Cedar Waxwing

Bombycilla cedrorum

\section{Fringillidae}

Golden-browed Chlorophonia

Chlorophonia callophrys

Yellow-throated Euphonia

Euphonia hirundinacea

\section{Passerellidae}

Chestnut-capped Brushfinch Arremon brunneinucha

\begin{tabular}{|c|c|c|c|c|c|}
\hline 0 & PMF & $\mathrm{R}$ & 2 & I & $\mathrm{LC}^{\mathrm{S}}$ \\
\hline 1 & PWF & $\mathrm{L}$ & 2 & I & $\mathrm{LC}^{\mathrm{I}}$ \\
\hline 0 & PMF & $\mathrm{L}$ & 2.5 & I & $\mathrm{LC}^{\mathrm{I}}$ \\
\hline 0 & PMF, PWF & $\mathrm{L}$ & 3 & I & $\mathrm{LC}^{\mathrm{I}}$ \\
\hline 0 & PMF, PWF & $\begin{array}{c}\mathrm{RR}, \\
\mathrm{L}\end{array}$ & 3 & I & $\mathrm{LC}^{\mathrm{U}}$ \\
\hline 0 & PMF & $\mathrm{R}$ & 3 & I & $\mathrm{LC}^{\mathrm{I}}$ \\
\hline 0 & PMF & $\mathrm{R}$ & 2.5 & I & $\mathrm{LC}^{\mathrm{U}}$ \\
\hline 0 & PMF & $\mathrm{R}$ & 2 & I & $\mathrm{LC}^{\mathrm{U}}$ \\
\hline 0 & NI & $\mathrm{R}$ & 3 & I & $\mathrm{LC}^{\mathrm{U}}$ \\
\hline
\end{tabular}

$\begin{array}{lccccccc}14 & \text { LMWF } & \text { E } & 1.5 & \text { I } & \text { CRWP } & \text { LC } / \text { End } & \text { SC, MR } \\ 0 & \text { PMF } & \text { R } & 2.5 & \text { I } & \text { LC } & \text { MR } \\ 1 & \text { LMWF } & \text { R } & 1 & \text { I } & \text { LC } & \text { MR } \\ 1 & \text { LMWF } & \text { R } & 1 & \text { I } & \text { LC } & \text { MR } \\ 0 & \text { PMF } & \text { L } & 2.5 & \text { I } & \text { LC } & \text { MR }\end{array}$

34 PMF, PWF,LMWF L 2 I $\quad$ LC $^{\mathrm{U}} \mathrm{M}, \mathrm{SC}, \mathrm{MR}$

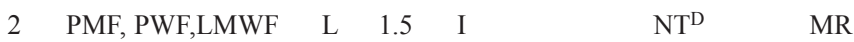

2 PWF,LMWF $\quad$ E $2 \quad$ I $\quad$ LC $^{\mathrm{D}} \quad \mathrm{SC}, \mathrm{MR}$

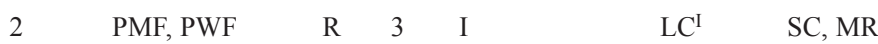

1 PMF, PWF $\quad$ R 2 I $\quad$ LC $^{\mathrm{D}}$ M, MR

$\begin{array}{llllllll}0 & \text { PWF } & \text { L } & 2.5 & \text { F } & \text { LC } & & \text { MR }\end{array}$

3 PWF $\quad$ E $2 \quad$ F $\quad$ CRWP LC $^{\mathrm{D} / \text { End }}$ SC, MR

2 PMF, PWF $\quad$ R $2.5 \quad$ F $\quad$ LC ${ }^{S} \quad$ SC, MR

$\begin{array}{lllllll}0 & \text { LMWF } & \mathrm{R} & 1.5 & \mathrm{G} & \mathrm{LC}^{\mathrm{D}} & \mathrm{MR}, \mathrm{MR}\end{array}$ 
CUADRO 2 (Continuación) / TABLE 2 (Continued)

Black-striped Sparrow

Arremonops conirostris

White-eared Ground-Sparrow

Melozone leucotis

Cabani's Ground Sparrow

Melozone cabanisi

Common Chlorospingus

Chlorospingus flavopectus

\section{Icteridae}

Montezuma Oropendola

Psarocolius montezuma

\section{Parulidae}

Ovenbird

Seiurus aurocapilla

LouisianaWaterthrush

Parkesia motacilla

Northern Waterthrush

Parkesia noveboracensis

Golden-winged Warbler

Vermivora chrysoptera

Black-and-white Warbler

Mniotilta varia

Tennessee Warbler

Oreothlypis peregrina

Kentucky Warbler

Geothlypis formosa

Blackburnian Warbler

Setophaga fusca

Chestnut-sided Warbler

Setophaga pensylvanica

Black-throated Green Warbler

Setophaga virens

Rufous-capped Warbler

Basileuterus rufifrons

Golden-crowned Warbler

Basileuterus culicivorus

Canada Warbler

Cardellina canadensis

Slate-throated Redstart

Myioborus miniatus

\section{Cardinalidae}

Scarlet Tanager

Piranga olivacea

Black-thighed Grosbeak

Pheucticus tibialis

Indigo Bunting

Passerina cyanea

\section{Thraupidae}

Blue-gray Tanager

Thraupis episcopus

Palm Tanager

Thraupis palmarum

$\begin{array}{cccccccc}1 & \text { PWF } & \text { R } & 2 & \text { G } & & \text { LC } & \text { MR } \\ 2 & \text { PMF, PWF, LMWF } & \text { R } & 2.5 & \mathrm{I} & & \text { LC } / \text { RPT } & \text { M, MR } \\ 0 & \text { PMF } & \text { R } & 2.5 & \text { G } & \text { CR } & \text { LC'/End } & \text { MR } \\ 1 & \text { LMWF } & \text { R } & 1.5 & \text { I } & & \text { LC }^{\mathrm{S}} & \text { MR }\end{array}$

$\begin{array}{llllllll}0 & \text { PMF } & \text { R } & 2.5 & \text { F } & \text { LC }^{\mathrm{S}} & \text { MR }\end{array}$

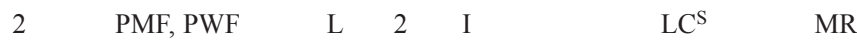

$\begin{array}{llllllll}0 & \text { LMWF } & \text { L } & 3 & \text { I } & \text { LC } & \text { MR }\end{array}$

$\begin{array}{llllllll}0 & \text { PWF } & \text { L } & 3 & \text { I } & \text { LC } & \text { MR }\end{array}$

$\begin{array}{llllllll}0 & \text { PWF } & \text { L } & 2.5 & \text { I } & \text { NT }^{\mathrm{D}} & \text { MR }\end{array}$

$\begin{array}{lllllll}\text { PWF } & \mathrm{L} & 2 & \mathrm{I} & \mathrm{LC}^{\mathrm{D}} & \mathrm{MR}\end{array}$

$\begin{array}{lllllll}0 & \text { PMF, PWF } & \text { L } & 2 & \text { I } & \text { LC }^{S} & \text { MR }\end{array}$

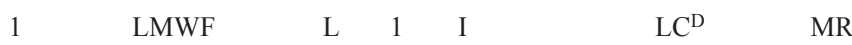

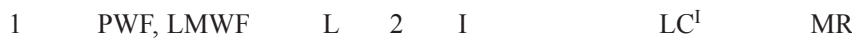

$\begin{array}{llllllll}1 & \text { PWF } & \text { L } & 2.5 & \text { I } & \text { LC }^{\mathrm{D}} & \text { MR }\end{array}$

$\begin{array}{lllllll}0 & \text { LMWF } & \text { L } & 2 & \text { I } & \text { LC } & \text { MR }\end{array}$

$\begin{array}{lllllll}0 & \text { PMF } & \text { R } & 2 & \text { I } & \text { LC }^{S} & \text { MR }\end{array}$

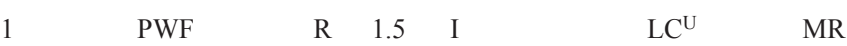

2 PWF, LMWF $\quad$ L $2.5 \quad$ I $\quad$ LC $\quad$ M, SC, MR

$\begin{array}{llllllll}1 & \text { PWF } & \text { R } & 2 & \text { I } & \text { LC } & \text { MR }\end{array}$

$\begin{array}{lllllll}0 & \text { PMF, PWF } & \text { L } & 3 & \text { I } & \text { LC } & \text { MR }\end{array}$

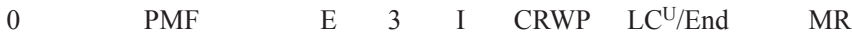

$\begin{array}{lllllll}0 & \text { PWF } & \mathrm{L} & 3 & \mathrm{G} & \text { LC }^{\mathrm{D}} & \mathrm{M}\end{array}$

$\begin{array}{lllllll}0 & \text { PMF } & \text { R } & 3 & \text { F } & \text { LC } & \text { MR }\end{array}$

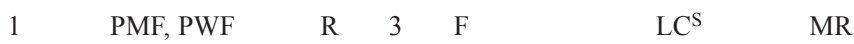




\begin{tabular}{|c|c|c|c|c|c|c|c|c|}
\hline \multicolumn{9}{|c|}{ CUADRO 2 (Continuación) / TABLE 2 (Continued) } \\
\hline $\begin{array}{l}\text { Spangle-cheeked Tanager } \\
\text { Tangara dowii }\end{array}$ & 0 & PWF & E & 2 & $\mathrm{~F}$ & CRWP & $\mathrm{LC}^{\mathrm{D}}$ & MR \\
\hline $\begin{array}{l}\text { Silver-throated Tanager } \\
\text { Tangara icterocephala }\end{array}$ & 2 & PMF, PWF, LMWF & $\mathrm{E}$ & 2 & $\mathrm{~F}$ & & $\mathrm{LC}^{\mathrm{D}}$ & $\mathrm{M}, \mathrm{MR}$ \\
\hline $\begin{array}{l}\text { Green Honeycreeper } \\
\text { Chlorophanes spiza }\end{array}$ & 0 & PWF & $\mathrm{E}$ & 2 & $\mathrm{~F}$ & & $\mathrm{LC}^{\mathrm{D}}$ & MR \\
\hline $\begin{array}{l}\text { Red-legged Honeycreeper } \\
\text { Cyanerpes cyaneus }\end{array}$ & 1 & PWF & $\mathrm{R}$ & 2 & $\mathrm{~N}$ & & $\mathrm{LC}^{\mathrm{S}}$ & $\mathrm{SC}, \mathrm{MR}$ \\
\hline $\begin{array}{l}\text { Yellow-faced Grassquit } \\
\text { Tiaris olivaceus }\end{array}$ & 3 & PMF, PWF & $\mathrm{R}$ & 3 & G & & $\mathrm{LC}^{\mathrm{I}}$ & MR \\
\hline $\begin{array}{l}\text { Buff-throated Saltator } \\
\text { Saltator maximus }\end{array}$ & 0 & PMF, PWF & $\mathrm{R}$ & 3 & $\mathrm{~F}$ & & $\mathrm{LC}^{\mathrm{D}}$ & MR \\
\hline
\end{tabular}

1. Taxonomy follows American Ornithologists' Union (AOU) (Chesser et al., 2016).

2. Life zones: premontane moist forest - PMF, premontane wet forest - PWF, Lower montane wet forest - LMWF (Fogden, 2014), NI - No information.

3. Migratory status: Resident no migrant - R, E - Altitudinal migrant, L -Latitudinal migrant, RR,L - Migrant with reproductive populations, based on Fogden (2014).

4. Forest dependence: Mature forest dependent -1 , occur in areas with at least $50 \%$ forest cover -2 , inhabit open areas -3 (Biamonte et al., 2011).

5. Trophic guild: C -Carnivore, F- Frugivore, G -Granivore, I -Insectivore, N -Nectarivore, O -Omnivore. (GonzálezSalazar et al., 2014).

6. Endemism: CRWP - Highlands of Costa Rica and West of Panama, CR - Costa Rica, CHP - Caribbean Honduras to Panama; EHWP - Eastern Honduras to West Panama based on (Garrigues et al., 2017).

7. Conservation Status: LC - Least Concern; NT - Near Threatened, D -Decreasing populations, S -Stable populations, I -Increasing populationsc/ End - Endangered, RPT - Reduced or Threatened Populations (IUCN, 2017; SINAC, 2017).

8. Sources of data: Museums - M; Survey Carcasses- SC, Monteverde Residents - MR.

not registered in the three life zones by Fogden (2014). The families with the most species represented were Parulidae (14), Trochilidae (13), Turdidae (10), and Tyrannidae (9).

Migratory status: Most collisions were by resident species, including 57 no migrants and 21 altitudinal migrants (Table 2).Twenty one (51\%) of 41altitudinal migrants documented by Fogden (2014) collided with windows.

Species and carcasses collected by method: Carcasses gathered by the Monteverde community resulted in 130 bodies of 46 species. Naturalists collected 81 bodies of 33 species. Photo reports generated data

TABLE 2

Number of species recorded colliding with windows and documented by migrant status, Monteverde, Costa Rica

\begin{tabular}{|c|c|c|}
\hline Migratory status & $\begin{array}{l}\text { Bird-window } \\
\text { collision species }\end{array}$ & $\begin{array}{l}\text { Birds documented in } \\
\text { Monteverde study area* }\end{array}$ \\
\hline Resident & 57 & 156 \\
\hline Altitudinal migrant & 21 & 41 \\
\hline Latitudinal migrant & 23 & 67 \\
\hline \multirow[t]{2}{*}{ Latitudinal migrant with reproductive populations } & 2 & 3 \\
\hline & 103 & 267 \\
\hline
\end{tabular}

* Species documented at premontane moist forest, premontane wet forest and lower montane wet forest line zones by Fogden (2014). 
TABLE 3

Number of species recorded colliding with windows and documented by forest dependence, Monteverde, Costa Rica

\begin{tabular}{ccc} 
Category of forest dependence & Bird-window collision species & Birds documented in Monteverde study area* \\
1 & 5 & 24 \\
1.5 & 19 & 41 \\
2 & 36 & 59 \\
2.5 & 21 & 54 \\
3 & 22 & 85 \\
& 0 & 4 \\
& 103 & 267 \\
\hline
\end{tabular}

* Species documented at premontane moist forest, premontane wet forest and lower montane wet forest line zones by Fogden (2014).

TABLE 4

Number of species recorded colliding with windows and documented by trophic guild, Monteverde, Costa Rica

\begin{tabular}{lcc}
\multicolumn{1}{c}{ Trophic guilds } & Bird-window collision species & Birds documented in Monteverde study area* \\
Insectivores & 57 & 157 \\
Frugivores & 22 & 35 \\
Nectarivores & 14 & 22 \\
Granivores & 10 & 24 \\
Omnivores & 0 & 4 \\
Carnivores & 0 & 20 \\
Aquatic invertebrates eaters & 0 & 3 \\
Scavengers & 0 & 2 \\
& 103 & 267 \\
\hline
\end{tabular}

* Species documented at premontane moist forest, premontane wet forest and lower montane wet forest line zones by Fogden (2014).

for 67 individuals of 41 species. Museums reported 22 individuals of 14 species, and the carcass survey allowed collecting 22 bodies of 14 species.

Forest dependence: We found different levels of forest dependence among the species that collided with windows. Most species $(35 \%)$ occur in areas with at least $50 \%$ of forest cover and just five species were classified as highly dependent on mature forest and secondary forest (1 and 1.5) (Table 3 ). In lower wet montane forest we found thirteen of 31 (42 $\%)$ species that collided as highly dependent on mature and secondary forest (1 and 1.5),13 (54\%) were also found to collide in the premontane wet forest; and only five $(21 \%)$ in the premontane moist forest (Table 1).
Feeding guilds: Collisions were mostly by insectivore species (57spp) (Table 4). Only $36 \%$ of the insectivore species documented by Fogden (2014) were found to collide. On the other hand, $64 \%$ of nectarivore and $63 \%$ of frugivore were documented to have collided with glass panes. Insectivores not documented in our study as window-kills include swallows, woodpeckers, and 28 species $(76 \%)$ of the Tyrannidae family (Fogden, 2014). Alternatively, the Olive-striped Flycatcher hit frequently in lower montane wet forest. No carnivores, scavengers, omnivores, nor birds that feed on aquatic macroinvertebrates were recorded as window casualties (Table 4).

Weight: Most collision species weighed less than 80 grams (84 spp.; $81 \%$ ). The 
TABLE 5

Number of species recorded colliding with windows in Monteverde, Costa Rica, per life zone and abundance status (Fogden, 2014)

\begin{tabular}{lccc}
\multicolumn{1}{c}{ Abundance Status } & Premontane moist forest & Premontane wet forest & Lower montane wet forest \\
Common & 15 & 20 & 18 \\
Fairly common & 13 & 16 & 5 \\
Uncommon & 9 & 12 & 1 \\
Rare & 1 & 5 & 5 \\
Vagrant & 0 & 3 & 0 \\
Status Uncertain & 1 & 0 & 0 \\
Not reported in the life zone & 10 & 8 & 2 \\
Total & 49 & 64 & 31 \\
\hline
\end{tabular}

smallest species were Canivet's Emerald (Chlorostilbon canivetii) $2.6 \mathrm{~g}$ and the Rubythroated Hummingbird $2.8 \mathrm{~g}$. The heaviest were Gray-headed Chachalaca (Ortalis cinereiceps) 500 g.; Keel-billed Toucan (Ramphastos sulfuratus) $500 \mathrm{~g}$.; Montezuma Oropendola (Psarocolius montezuma) 520 g.; and the Black Guan (Chamaepetes unicolor) $950 \mathrm{~g}$. Most of the birds are small or medium sized. Neither Accipitridae family species documented in the area $(\mathrm{n}=15, \mathrm{Mdn}=480 \mathrm{~g})$ were found to collide with windows, nor Cathartidae family species $(\mathrm{n}=2, \operatorname{Mdn}=1600 \mathrm{~g})$.

Abundance per life zone: More species collided with windows in premontane wet forest than in premontane moist forest or in lower montane wet forest (Table 5). Nine species were found in the three life zones, 21 only in premontane moist forest, 32 only in premontane wet forest, and 16 only in lower montane wet forest (Table 1). Most victims in all life zones were classified as Common and Fairly Common, but among the affected were also species previously classified as Uncommon, Rare, Vagrant, and others not reported by Fogden (2014) in the life zones (see Table 5).

Endemism, population trend, and species vulnerability status: We found 12 endemic species colliding with windows, four of them with declining populations (Garrigues et al., 2017). Thirty-six species were recorded having declining populations while 34 are considered as stable populations; 18 increasing, and the population trend for 15 species is unknown (IUCN, 2017). According to the IUCN Red List (2017), most collision species are classified in the category of Least Concern (97 spp.; $94 \%$ ) and five as Near Threatened, and one Vulnerable (Table 1). Six species are on the List of Endangered Species of the National System of Conservation Areas of Costa Rica and five on the list of reduced populations or threatened species for Costa Rica (SINAC, 2017) (Table 1). The populations of six endemic species are considered to be decreasing or unknown and are on the list of endangered species of Costa Rica: Black-thighed Grosbeak (Pheucticus tibialis), Three-wattled Bellbird, Black Guan, Black-faced Solitaire, Golden-browed Chlorophonia, and Orange-bellied Trogon (Table 1).

\section{Species that collide most frequently with windows:}

A) Expert interviews. Five resident biologists interviewed cited Swainson's Thrush as the migratory species that collides with windows in higher numbers than other species in the area, mainly during April-May and September-October. Three of the five interviewees stated that, among the resident species, the Northern Emerald-Toucanet collides most often, the other two judged Clay-colored Thrush (Turdus grayi), pigeons, and hummingbirds, Long-tailed Manakin, and Keel-billed Toucan.

B) Community survey. A total of 58 residents answered the survey, one per house in 
premontane wet forest and lower montane wet forest. Forty-three surveyed residents (74\%) could recall a bird hitting their home windows at least once. Of these, the Northern EmeraldToucanet was described $(n=16)$ as the most common casualty, followed by hummingbirds $(\mathrm{n}=15)$, and pigeons $(\mathrm{n}=9)$. After showing pictures of eight species, $35(60 \%)$ householders recognized and reported that Swainson's Thrushes collided with windows, and 34 (59 \%) reported hummingbirds and Northern Emerald-Toucanets.

C) Museum records. Data from the National Museum of Costa Rica (NMCR) consisted of 14 window-killed specimens of seven species from Monteverde; of that total, eight specimens $(57 \%)$ were Swainson's Thrushes (Table 1). Eight window-killed specimens of seven species were in the collection of the Zoology Museum of the University of Costa Rica: Silver-throated Tanager (Tangara icterocephala), Mountain Elaena (Elaenia frantzii), Scaled Antpitta (Grallaria guatimalensis), Purple-throated Mountain-gem Lampornis calolaemus, Olivestriped Flycatcher (Mionectes olivaceus), Graythroated Leaftosser (Sclerurus albigularis), and two Northern Emerald-Toucanet specimens, including a specimen collected by Gary Stiles in 24 December 1978.

D) Naturalists. Eighty-one frozen specimens (31 species) collected prior to this research and stored in freezers were shared by naturalists (biologists and people interested in nature) of Monteverde. Date of collection was not always available, but the oldest specimens with information dated from 2008. Twentyeight specimens were collected in lower montane wet forest, 45 in premontane wet forest and eight did not have information about the place of collection. The most abundant species was Swainson's Thrush $(\mathrm{n}=16)$, followed by Blackfaced Solitaire $(\mathrm{n}=10)$, Northern Emerald Toucanet $(n=7)$, and Long tailed Manakin $(n=7)$.

Carcass surveys: From October 2014 to August 2016 a total of 152 carcasses consisting of 50 species classified in 15 families were collected by at least 63 volunteers $(n=130$ carcasses) and RMMO $(\mathrm{n}=22)$ in Monteverde between 4 October 2014 and 29 August 2016 in premontane wet forest and lower montane wet forest. One hundred and six bodies (70 \%) were collected in premontane wet forest and $45(30 \%)$ in lower montane wet forest. The most common species were Swainson's Thrush $(\mathrm{n}=34)$, Northern Emerald-Toucanet $(\mathrm{n}=14)$, Black-faced Solitaire (Myadestes melanops) $(\mathrm{n}=14)$, and the Olive-striped Flycatcher( $\mathrm{n}=$ 10). The families with the highest strike rates were Turdidae $(\mathrm{n}=57)$, Trochilidae $(\mathrm{n}=23)$, Tyrannidae $(\mathrm{n}=16)$, and Ramphastidae $(\mathrm{n}=$ 14). We were not able to identify two carcasses to the species level.

The most common species collected in premontane wet forest was the Swainson's Thrush $(\mathrm{n}=26)$ and the Northern Emerald-Toucanet $(\mathrm{n}=14)$, whereas the most common species collected in lower montane wet forest was the Black-faced Solitaire $(n=14)$, followed by Swainson's Thrush $(\mathrm{n}=6)$, and Olive-striped Flycatcher $(n=6)$. Ten $(71 \%)$ of 14 Blackfaced Solitaire were obtained from 24 June to July 25, 2015 in lower montane wet forest.

In relation to age and sex, 75 (49\%) of window casualties were mature, 60 (39.5\%) immature, and 17 (11\%) age unknown. For specific species, Northern Emerald-Toucanet specimens consisted of five (36\%) immatures and nine (64\%) matures; the number of casualties were equal for Black-faced Solitaire with six (50\%) immatures, six (50\%) matures; Swainson's Thrush consisted of 19 (56\%) matures, 11 (32\%) immatures; and four unknown $(12 \%)$.

For all individuals of all species combined, 59 were males, 33 females, and 60 unknown. For Swainson's Thrush we identified seven females, nine males, and 18 unknown. For Northern Emerald-Toucanets, both sexes were equally represented with six males, six females, and two unknown. For the Black-faced Solitaire we counted seven males, two females, and five unknown.

In relation to migratory status, 62 (41\%) were altitudinal migrants, 51 latitudinal migrants $(34 \%)$, and 37 residents $(25 \%)$. We 
found an increase in the number of long distance migrants colliding in April $2015(\mathrm{n}=17)$ and October $2015(\mathrm{n}=10)$ (Fig. 2). Fifteen (88 $\%)$ of the long-distance migrants in April were Swainson's Thrush, the other two were Wood Thrush and a Ruby-throated Hummingbird. In October, eight of the migratory species were Swainson's Thrush and the other two were Black-and-white Warbler and Yellow-throated Vireo (Vireo flavifrons).

The number of resident species colliding with windows increased in June and July. The number of immature residents collected increased only slightly in June (nine immature, seven mature), but more so in July (11 immature, five mature) and August (seven immature, 0 mature) (Fig. 3.)

\section{DISCUSSION}

A substantial number of bird species known to occur in Monteverde were documented as victims of bird-window collisions. Even though the number of species recorded in this study is much higher than the 57 species registered in Reserva Particular do Patrimônio Natural Santuário do Caraça, Minas Gerais, an Important Bird Area in Brazil (Santos et al., 2017) and 25 species in a rural area in Colombia (Ocampo-Peñuela, Peñuela-Recio, \& OcampoDurán, 2015), it likely underestimates the true number. Some of the factors for the presumed underestimation of our results are: a) in museums, it is not mandatory for the specimen tag to indicate the cause of death of birds. b) With the use of the citizen science approach, participants may not have been aware of or interested in this study. Participation could have been affected by motivation (Cooper, Dickinson, Phillips, \& Bonney, 2007), time available to search for and to report dead birds, cost of taking the carcasses to the different storage freezers, and the long time frame of the study. Even when participants were highly motivated, they could have failed to hear a bird striking a window or could have been absent during the collision event (Bracey, Etterson, Niemi, \& Green, 2016). Despite this, citizen science was crucial for gathering data and also probably increased awareness and knowledge about this subject (Chandler et al,

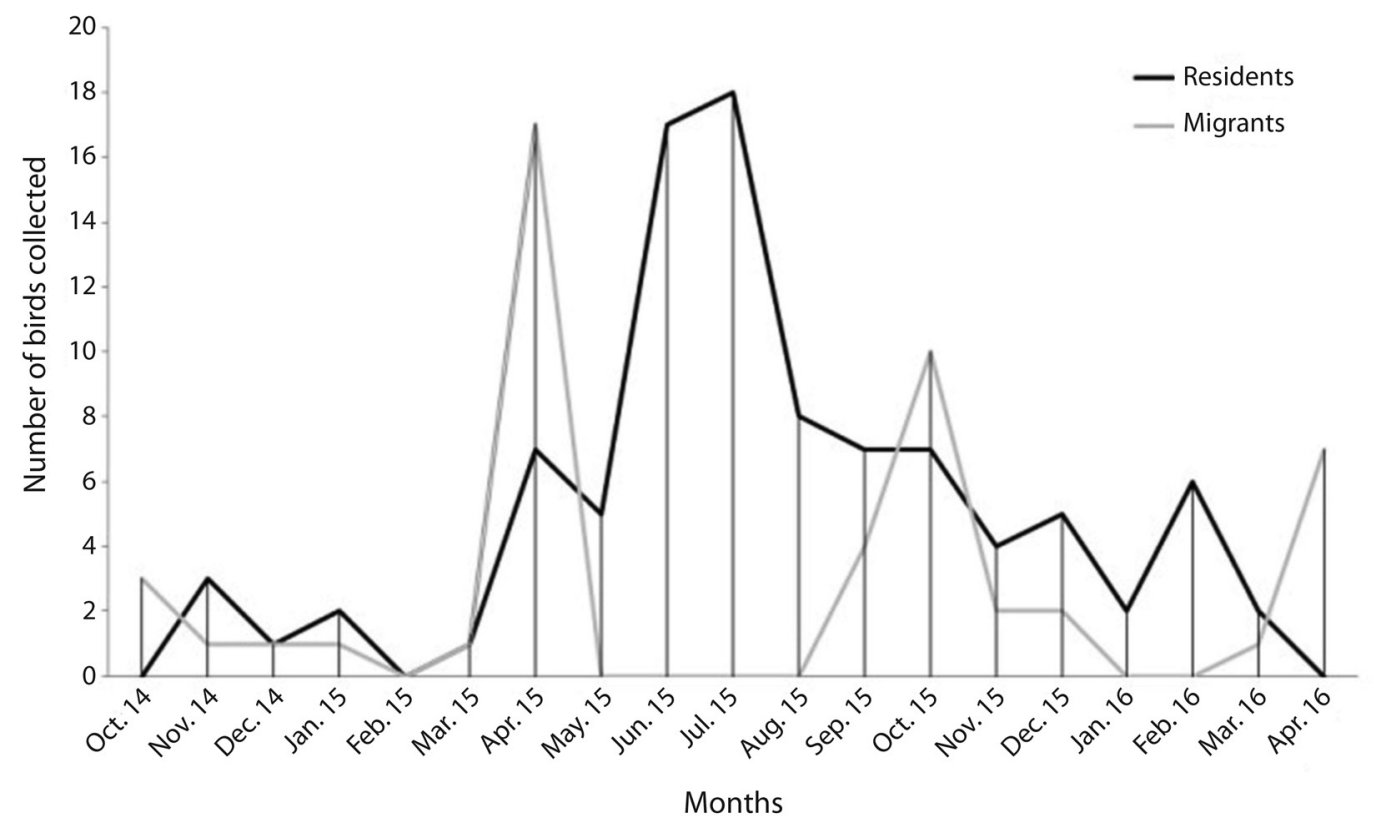

Fig. 2. Number of window-killed birds collected by month and migratory status in Monteverde, Costa Rica (from October 2014 to April 2016, $\mathrm{n}=145$ ). 


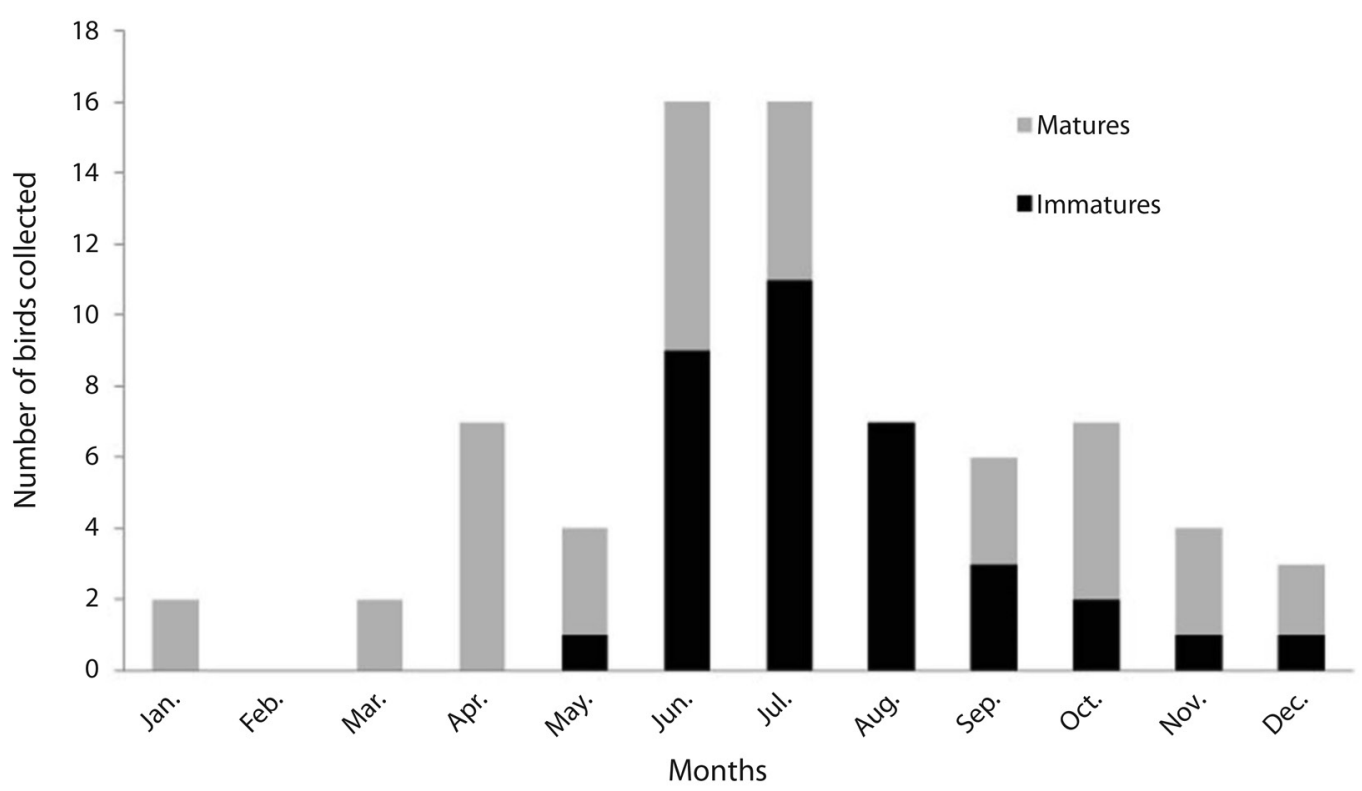

Fig. 3. Number of window-killed resident birds collected by month and age in Monteverde, Costa Rica (from January 2015 to December 2015).

2017; Cooper et al., 2007). c) A further factor that could have affected bird collisions detectability was the removal of the bird bodies by scavengers, such as coatis (Nasua narica) and domestic cats (Felis domesticus). Also, carcasses of birds that fell into vegetation, or injured birds that moved before dying, would also go undetected (Bracey et al., 2016).

We found that most species affected by window collisions in the study area were residents. Santos et al. (2017) and OcampoPeñuela et al. (2015), also found mostly resident species in their studies in rural conditions in the Neotropics. It is possible that the high density of year-round resident birds of Monteverde, and the deception caused by reflection of vegetation in windows, causes the high mortality of resident individuals (Gelb \& Delacretaz, 2009; Klem et al., 2009; Borden \&Lockhart, 2010). Since both juveniles and adults were found among the victims, we think birds are unable to learn to avoid the danger, as Klem (1989) also concluded.

We documented window-kills in endemic Neotropical families, including guans
(Cracidae), toucans (Ramphastidae), motmots (Momotidae), and manakins (Pipridae). Ocampo-Peñuela et al. (2016) and Santos et al. (2017) also found species of these families in their studies in rural areas in the Neotropics. The Northern Emerald-Toucanet was cited as one of the species most often reported as victim of window collisions.

As Loss et al. (2014) described for the United States, warblers, hummingbirds, and thrushes also were found to be vulnerable in Monteverde. The families Parulidae and Trochilidae had the highest number of species affected. Menacho-Odio (2015) found that hummingbirds are frequently reported as window casualties in Costa Rica. Klem (2014) mentioned that any attractant to the vicinity of the windows increases fatalities. It is likely that the presence of flowering plants and feeders in home and institutional gardens attract hummingbirds, thus increasing their risk of hitting glass in the buildings. In this case, incentives, like the Costa Rican government's Certification for Tourism Sustainability, that promote planting vegetation to attract resident 
and migratory birds around hotels should be reevaluated. Simultaneously, methods preventing window collisions should be promoted (Instituto Costarricense de Turismo, 2013). Hummingbird behaviors such as high-speed flight, male territoriality and associated aggression, may also increase the risk of collisions (Graham, 1997). Artificial feeders are common in Monteverde and in many other touristic areas of Costa Rica. In Costa Rica it is illegal to feed wildlife; however, this regulation is not enforced so it is important to communicate to residents and managers of lodges that feeders should be located less than one meter from any glass surface (Klem, 2014). Most of the Parulidae species found are migrants; among species extremely vulnerable to building collisions (Loss et al., 2014), all of which we found window killed in Monteverde, include Golden-winged Warbler (Vermivora chrysoptera), Canada Warbler (Cardellina canadensis), and Kentucky Warbler (Geothlypis formosa). In their study, Loss et al. (2014) pointed out that, for these three species, building collisions may contribute to their population decline. In our study, we found that buildings in rural areas may also be affecting bird populations during migration.

Swainson's Thrush and Black-faced Solitaire, two species found to collide with windows in high numbers in Monteverde, belong to the Turdidae family. In Colombia, OcampoPeñuela et al. (2016) found that the Blackbilled Thrush (Turdus ignobilis) was one of the two species with highest collision frequency. Klem (2014) described how birds such as hummingbirds, ovenbirds, and thrushes regularly fly through restricted passageways in dense vegetation, making them more susceptible to hit windows frequently. Although neither the expert interviews nor community survey indicated that the Black-faced Solitaire was killed in large numbers, we found a relatively high number of individuals of this species colliding with buildings in the lower montane wet forest. Since most people we interviewed do not live in the lower montane wet forest, we suspect that many window related deaths of the
Black-faced Solitaire were overlooked. For this reason, we can expect that more urbanization in lower montane wet forest will increase the mortality of the Black-faced Solitaire, a species considered Vulnerable in Costa Rica due to the popular practice of capturing and keeping them as pets in cages (Stiles \& Skutch, 2003; Arévalo, 2010; Oviedo \& Menacho-Odio, 2013).

Most species that collided with windows in our study were insectivores, the trophic guild with the most species in Monteverde (Young \& McDonald, 2014). However, the number of insectivores killed was small, based on the total number of species in this trophic guild. Insectivores not documented as window-kills include swallows, woodpeckers, and many species of the Tyrannidae family (Fogden, 2014). Alternatively, the Olive-striped Flycatcher hit frequently in lower montane wet forest.

Among the omnivore species, the Brown Jay (Psilorhinus morio) is considered common in Monteverde, but there was no evidence of collisions for this specie. Kahle, Flannery, \& Dumbacher (2016) mentioned that flock species may be underrepresented because it may easier for them to detect windows and signal to others.

Although we did not find carnivorous and omnivorous species as windows casualties, they are recorded as victims in other studies (Menacho-Odio, 2015; Kahle et al., 2016). Based on this, we believe that with an increased sampling effort, birds of these trophic guilds would be likely recorded striking windows in Monteverde.

The number of window collision casualties considered Endangered, Near Threatened, Vulnerable, and Endemic species demonstrates cause for conservation concern. Thus the increasing number of buildings and the preference for large panes of glass in residences and tourist structures (Menacho-Odio, 2018), should be monitored and regulated to reduce avian mortality. It is also necessary to focus attention on species such as the Orange-bellied Trogon, Black Guan, Three-wattled Bellbird, the Golden-browed Chlorophonia and other 
Neotropical endemic species whose populations are decreasing.

Twenty-one altitudinal migrant species were recorded as casualties in this study. Window collision risk is predicted to increase as birds move to lower more urbanized areas during their migratory passage. The Threewattled Bellbird, the Black-faced Solitaire, the Resplendent Quetzal, and the Golden-browed Chlorophonia are known window casualties that are altitudinal migrants. Our results document window strikes by two juvenile Threewattled Bellbirds; one (survived) in Los Llanos and another (died) in Monteverde (premontane wet forest), during June and July 2015 (Fig. 1). An average of 90 Three-wattled Bellbirds were counted in the last six years in Monteverde (Sandoval, 2014), making every individual killed in a window a significant loss to the local population (Debra Hamilton, pers. comm., 2015.).

The expected increase in the number of buildings in Monteverde is anticipated to intensify the risk of bird-window collisions, as occurred in the premontane wet forest in our study area. Currently, Costa Rica Wildlife Conservation Law (SINAC, 2017) does not include any regulation for preventing bird-window related deaths. Moreover, there are no regulations in Monteverde to prevent an increase in urbanization. Consequently, window-kills are expected to become an increasing threat to the local avifauna. Education and research for collision prevention will be required for conservation, economic, legal, and ethical planning to avoid the unwanted losses of the birds that more and visitors come to Monteverde to see (Klem, 2014). For example, there is a need to promote the use of effective methods to prevent bird-window collisions and to discourage the use of non-effective large panes of glass on new buildings.

Future studies should address the estimated or, ideally, actual abundance of specific species in and around human dwellings, and the use of more rigorous protocols by volunteers, as suggested by Loss, Will, \& Marra (2015), to obtain a more comprehensive understanding.
It is essential to include the monitoring of hotels and other commercial buildings in future investigations, given risks these specific structures, with their large areas of sheet glass, pose to birds.

Bird-window collisions affect a high number of species of birds in Monteverde. Most window-killed species are residents, some endemics, threatened with declining populations. Seeking a solution to protect birds from windows should be an important goal for this IBA and the rest of Costa Rica.

We suggest that additional research should include a greater proportion of the population of this area in order to increase concern and knowledge about this important conservation issue for birds and people, especially among commercial interests such as hotel owners and others employed in the birding-related industry. Additionally, more effort should be given to areas where high diversities of birds coexist with buildings boasting large surface area in windows. This study reinforces the importance of public participation (citizen science) for achieving a comprehensive understanding of the problem.

Ethical statement: authors declare that they all agree with this publication and made significant contributions; that there is no conflict of interest of any kind; and that we followed all pertinent ethical and legal procedures and requirements. All financial sources are fully and clearly stated in the acknowledgements section. A signed document has been filed in the journal archives.

\section{ACKNOWLEDGMENTS}

We are grateful to D. Klem Jr. for his valuable advice. L. Moreno, F. Joyce, K. Van Dusen, D. Hamilton, K. Master, A. Gora, R. La Val, A. Pounds, and M. Tandy helpfully contributed prior to and during the study. Monteverde Institute, Monteverde Cloud Forest Reserve of the Tropical Science Center, Monteverde Biological Station, and the Monteverde Friends School not only provided space for 
gathering bodies of dead birds, but also the collaboration of their staff and researchers for searching for carcasses of birds. M. Leiton and A. Gora helped during the study to gather the carcasses of birds that staff and neighbors provided. Monteverde residents informed and helped with this research, especially C. Acuña, L., B. and R. Guindon, R. Solano, A. Pounds, M. Guindon, N. Solano, G. Bello, G. Cisneros, M. Rockwell, S. and R. Montiel, E. Fuentes, L. Trejos, K. Nishida, J. Chaves, D. Joslin, R. Alvarado, and H. Whitfield. A. Robledo and E. Navarro assisted with the survey. R. Chinchilla and B. Alvarez Garay helped with SIG and maps; L. Bermudez, F. Bolaños and G. Avalos helped with advice to improve the article. The National Museum of Costa Rica and the Zoology Museum of the University of Costa Rica provided information and gathered many specimens collected. R. Garrigues, D. Martinez, J. Chavez and D. Quesada helped with some species identification. R. and J. May and N. Arcilla provided useful inputs in the English usage of this manuscript. R. and DOCINADE professors provided valuable advice.

\section{RESUMEN}

Ecología, endemismo y estatus de conservación de aves que han colisionado contra ventanas de vidrio en Monteverde, Costa Rica. Cada año, a nivel global, mueren miles de millones de aves al colisionar contra ventanas. A pesar de ello, pocos registros documentan colisiones aves-ventanas en zonas altamente diversas en el Neotrópico. En este estudio, nosotros documentamos especies que colisionaron en ventanas en Monteverde, Costa Rica y describimos su estatus ecológico y de conservación. Nosotros obtuvimos información de distintas fuentes, incluyendo datos de registros de museos y de observaciones de residentes de Monteverde que participaron como "científicos ciudadanos" entre mayo 2014 y diciembre 2017. Nosotros realizamos búsquedas de cuerpos de aves entre marzo del 2015 y febrero 2016. Clasificamos especies que hubieran golpeado de acuerdo a su estatus de migración, dependencia del bosque, gremio alimentario, peso, abundancia, endemismo y de conservación. Nosotros registramos 103 especies que habían golpeado ventanas en Monteverde (98 de 267 especies documentadas en tres zonas de vida y cinco no registradas en el área). Muchas especies víctima de colisiones se caracterizan por ser residentes, pequeños, insectívoros, considerados comunes o bastante comunes, con poblaciones decrecientes. Las familias más representadas son Parulidae (14 spp.), Trochilidae (13 spp.), Turdidae (10 spp.) y Tyrannidae ( 9 spp.). La mayoría de las especies son paseriformes (Orden Passeriformes) (71 spp.). Ni gavilanes ni zopilotes golpearon en edificios durante nuestro estudio. Las tres especies más comúnmente muertas por ventanas fueron el Zorzal de Swainson (Catharus ustulatus), el tucancillo verde (Aulacorhynchu sprasinus) y el jilguero (Myadestes melanops). Entre las especies afectadas cinco especies tienen estatus de Casi Amenazadas y una Vulnerable según la Lista Roja de la UICN, incluyendo el Quetzal (Pharomachrus mocinno) y los pájaros campana (Procnias tricarunculatus). Cinco especies están en lista de peligro de extinción y cuatro con poblaciones reducidas según la lista del Sistema Nacional de Áreas de conservación de Costa Rica (SINAC). 12 especies endémicas fueron víctimas de colisiones. El bosque premontano húmedo es la zona de vida donde más especies fueron encontradas $(n=64$ spp.), seguidos por el bosque premontano muy húmedo y el bosque muy húmedo montano bajo $(\mathrm{n}=31 \mathrm{spp}$.). Estos hallazgos demuestran la urgente necesidad de implementar medidas de conservación para mitigar la mortalidad de las aves originada por colisión contra ventanas. El aplicar métodos para proteger a las aves de las ventanas debe ser una importante meta para esta AICA así como para el resto de Costa Rica. Recomendamos desarrollar acciones educativas, legales y continuar recolectando información, tanto en Monteverde como en otras áreas de Costa Rica para aumentar el conocimiento acerca de la colisión de aves con ventanas.

Palabras clave: mortalidad de aves; especies amenazadas; colisiones en paneles de vidrio; zonas de vida; vulnerabilidad a colisiones; ciencia ciudadana; Monteverde, Bosque Nuboso Tropical.

\section{REFERENCES}

Agudelo-Álvarez, L. (2006). Colisión de aves contra los ventanales del campus de la Universidad Javeriana, Sede Bogotá. Alternativas de mitigación. Retrieved from http://www.javeriana.edu.co/ear/fac/documents/ colisionaves.pdf

Agudelo-Álvarez, L., Moreno-Velasquez, J., \& Ocampo-Peñuela, N. (2010). Colisiones de Aves contra ventanales en un campus universitario de Bogotá, Colombia. Ornitología Colombiana, 10, 3-10.

Arévalo, J. E. (2010). Evaluación de las aves silvestres mantenidas en cautiverio en comunidades cercanas al Volcán Poas, Costa Rica. Zeledonia, 14(1), 1-11.

Biamonte, E., Sandoval, L., Chacón, E., \& Barrantes, G. (2011).Effect of urbanization on the avifauna in a tropical metropolitan area. Landscape Ecology, 26, 183-194. DOI: 10.1007/s10980-010-9564-0 
Bird Life International.(2017). Data Zone.Global IBA Criteria. Retrieved from http://datazone.birdlife.org/ site/ibacritglob

Borden, C., \& Lockhart, O. (2010). Seasonal, Taxonomic, and Local Habitat Components of Bird-window Collisions on an Urban University Campus in Cleveland, OH. Ohio Journal of Science, 110(3), 44-52.

Bracey, A. M., Etterson, M. A., Niemi, G. J., \& Green, R. F. (2016).Variation in bird-window collision mortality and scavenging rates within an urban landscape. The Wilson Journal of Ornithology, 128(2), 335-367.

Briske, T., Campos-Silva, 1., \& Piratelli, A. (2017).Relationship between bird-of-prey decals and bird-window collisions on a Brazilian university campus. Zoología, 34, 1-8. DOI:10.3897/zoologia.34.e13729

Chandler, M., See, L., Copas, K., Bonde, M. A., Claramunt López, B., Danielsen, F., ...Turak, E. (2017). Contribution of citizen science towards international biodiversity monitoring. Biological Conservation, 213(Part B), 280-294.

Chesser, T., Burns, K., Cicero C., Dunn J., Kratter A., Lovette I., ... Winker K.(2016). Fifty-seventh supplement to the American Ornithologists' Union Checklist of North American Birds, 133, 544-560. DOI 10.1642/auk-16-77.1

Cooper, C. B., Dickinson, J., Phillips, T., \& Bonney, R. (2007). Citizen science as a tool for conservation in residential ecosystems. Ecology and Society, 12(2), 11. Retrieved from http://www.ecologyandsociety. org/vol12/iss2/art11/

Cupul-Magaña, F. (2003). Nota sobre colisiones de aves en las ventanas de edificios universitarios en Puerto Vallarta, México. Huitzil, 4(2), 17-21.

Cusa, M., Jackson, D. \& Mesure, M. (2015). Window Collisions by migratory bird species: urban geographical patterns and habitat associations. Urban Ecosystems, 18(4), 1427-1146. DOI:10.1007/s11252-015-0459-3

Davis, J. (2009). The Creation and Management of Protected Areas in Monteverde, Costa Rica. Global Environment, 3, 96-119.

Fogden, M. (2014). Apéndice 9. Aves de la zona de Monteverde. In N. T. Wheelwright \& N. M. Nadkarni (Eds.), Monteverde: Ecología y conservación de un bosque nuboso tropical (pp.831-842). New York: Bowdoin's Scholars' Bookshelf. Retrieved from http://digitalcommons.bowdoin.edu/scholars-bookshelf/3/

Garrigues, R. (2014). The Birds of Costa Rica. A Field Guide. Ithaca. NY: Cornell University Press.

Garrigues, R., Araya-Salas, M., Camacho-Varela, P., Montoya, M., Obando-Calderón, G., \& Ramírez-Alán, O. (2017). Lista Oficial de las Aves de Costa RicaActualización 2017. Comité de especies raras y registros ornitológicos de Costa Rica (Comité Científico). Costa Rica: Asociación Ornitológica de Costa Rica. Retrieved from https://istaoficialavesdecostarica.wordpress.com/lista-oficial/lista-oficial-online/

Gelb, Y., \& Delacretaz, N. (2009). Window and Vegetation: Primary Factors in Manhattan Bird Collisions. Northeastern Naturalist, 16(3), 455-470.

Gómez-Moreno, V., Herrera-Herrera, J., \& Niño-Maldonado, S. (2018). Colisión de aves en ventanas del Centro Universitario Victoria, Tamaulipas, México. Huitzil, Revista Mexicana de Ornitología, 19(2), 227 236. DOI: $10.28947 /$ hrmo.2018.19.2.347

González-Salazar, C., Martínez-Meyer, E., \& López-Santiago, G. (2014). A hierarchical classification of trophic guilds for North American birds and mammals. Revista Mexicana de Biodiversidad, 85, 931-941. DOI: $10.7550 / \mathrm{rmb} .38023$

Graham, D. (1997). Spider webs and windows as potentially important sources of hummingbird mortality. Journal of Field Ornithology, 68(1), 98-101.

Hager, S., \& Cosentino, B. (2014).Surveying for bird carcasses resulting from window collisions: a standardized protocol. Peerj Preprints. DOI:10.7287/peerj. preprints.406v1

Hager, S. B., \& Craig, M. E. (2014).Bird-window collisions in the summer breeding season. PeerJ, 2:e460. DOI: $10.7287 /$ peerj.preprints.407v1

Holdridge, 1. (1967). Life Zone Ecology. San José, Costa Rica: Tropical Science Center.

Instituto Costarricense de Turismo. (2013). Certificación para la Sostenibilidad Turistica (CST). Normas CST para empresas de hospedaje. Costa Rica. Retrieved from http://www.turismo-sostenible.co.cr/pdf/ norma_cst_hoteles_costa_rica.pdf

IUCN. (2017). The IUCN Red List of Threatened Species. Retrieved from http://www.iucnredlist.org/

Kahle, L. Q., Flannery, M. E., \& Dumbacher, J. P. (2016). Bird-window collisions at a west-coast urban park museum: analyses of bird biology and window attributes from Golden Gate Park, San Francisco. PLoS one, 11(1), e0144600.DOI: 10.1371/journal. pone. 0144600

Kappelle, M. (2016). Costa Rican Ecosystems. Chicago: University of Chicago Press.

Klem Jr, D.(1989). Bird-Window Collisions. Wilson Bulletin, 101(4), 606-620.

Klem Jr, D. (2014). Landscape, legal, and biodiversity threats that windows pose to birds: a review of an important conservation issue. Land, 3(1), 351-361. 
Klem Jr, D. (2015). Bird-window collisions: A critical animal welfare and conservation issue. Journal of Applied Animal Welfare Science, 18 (sup1), s11-s17. DOI:10.1080/10888705.2015.1075832

Klem Jr, D., Farmer, C. J., Delacretaz, N., Gelb, Y., \& Saenger, P. G. (2009). Architectural and landscape risk factors associated with bird-glass collisions in an urban environment. The Wilson Journal of Ornithology, 121(1), 126-134.

Kummer, J. A., Bayne, E. M., \& Machtans, C. S. (2016). Use of citizen science to identify factors affecting bird-window collision risk at houses. The Condor: Ornithological Applications, 118(3), 624-639.

Loss, S. R., Will, T., Loss, S. S., \& Marra, P. P. (2014). Bird-building collisions in the United States: Estimates of annual mortality and species vulnerability. Condor, 116, 8-23.

Loss, S. R., Will, T. \& Marra, P. P. (2015). Direct mortality of birds from anthropogenic causes. Annual Review of Ecology, Evolution and Systematics, 46, 99-120.

Machtans, C. S., \& Thogmartin, W. E. (2014).Understanding the value of imperfect science from national estimates of bird mortality from window collisions. Condor: Ornithological Applications, 116, 3-7.

Machtans, C.S., Wedeles C.H., \& Bayne, E. (2013). A first estimate for Canada of the number of birds killed by colliding with building windows. Avian Conservation Ecology, 8(2), 6.

Menacho-Odio, R.M. (2015). Colisión de aves contra ventanas en Costa Rica: conociendo el problema a través de datos de museo, ciencia ciudadana y el aporte de biólogos. Zeledonia, 19(1),10-21.

Menacho-Odio, R.M. (2018). Local perceptions, attitudes, beliefs and practices toward bird-window collisions in Monteverde, Costa Rica. UNED Research Journal, 10(1), 33-40.

Nadkarni, N., \& Wheelwright, N. (2014). Introducción. In N. Wheelwright, \& N. Nadkarni (Eds.), Monteverde: ecología y conservación de un bosque nuboso tropical (pp. 1-48). New York: Oxford University Press. Retrieved from http://digitalcommons.bowdoin.edu/ scholars-bookshelf/3/

Obando-Calderón, G., Chaves-Campos, J., Garrigues, R., Martínez-Salinas, A., Montoya, M., Ramirez, O., \& Zook, J. (2017). Lista oficial de especies endémicas para la región. Costa Rica: Asociación Ornitológica de Costa Rica. Retrieved from https:// listaoficialavesdecostarica.wordpress.com/ lista-oficial/aves-endemicas-de-costa-rica/

Ocampo-Peñuela, N., Peñuela-Recio, L., \& OcampoDurán, Á. (2015). Decals prevent bird-window collisions at residences: a successful case study from Colombia. Ornitología Colombiana, 15, 84-91.

Ocampo-Peñuela, N., Winton, R., Wu, C., Zambello, E., Wittig, T., \& Cagle, N. (2016). Patterns of birdwindow collisions inform mitigation on a university campus. PeerJ, 4, e1652. DOI: 10.7717/peerj.1652

Oviedo, P., \& Menacho-Odio, R. (2013). Situación de los jilgueros (Myadestes melanops) en cautiverio en los alrededores del Parque Nacional Tapantí, Costa Rica. Zeledonia, 17(1), 54-61.

Oviedo, S., \& Menacho-Odio, R. M. (2015). Actitud en la preferencia de métodos para evitar el choque de aves contra puertas y ventanas de vidrio en Costa Rica. Zeledonia, 19, 22-31.

Sandoval, L. (2014). Informe de los seis años del censo nacional de Pájaros Campana (Procnias tricarunculatus) en Costa Rica. Costa Rica: Unión de Ornitólogos. Retrieved from http://uniondeornitologos.com/ wp-content/uploads/2014/12/informe_del_censo_ nacional_de_campanas_6-anos.pdf

Sánchez, J., Criado, J., Sánchez, C.,\& Sandoval, I. (2009). Costa Rica. In Devenish et al. (Eds.), Important Bird Areas Americas - Priority sites for biodiversity conservation (pp. 149-156). Quito: Birdlife International.

Santos, L., Ferreira, V., \& Ferreira, M. (2017).Bird mortality due to collisions in glass panes on an Important Bird Area of southeastern Brazil. Revista Brasileira de Ornitologia, 25(2), 90-101.

SINAC. (2017). Listado de especies de fauna silvestre en peligro de extinción y listado de especies de fauna silvestre con población reducida o amenazada. Retrieved from http://www.pgrweb.go.cr/scij/Busqueda/ Normativa/Normas/nrm texto completo.aspx?param $1=$ NRTC \&nValor $1=1 \& \bar{n}$ Valor $2=84908 \&$ nValor $3=10$ 9703\&strTipM=TC

Stiles, F. G., \& Skutch, A. F. (2003). Guía de Aves de Costa Rica. Santo Domingo de Heredia: Instituto Nacional de Biodiversidad.

Young, B., \& McDonald, D. (2014). Aves. In N. Wheelwright \& N. Nadkarni (Eds.), Monteverde: ecología y conservación de un bosque nuboso tropical. New York: Oxford University Press. Retrieved from http:// digitalcommons.bowdoin.edu/scholars-bookshelf/3/ 